\title{
ON THE EQUIFORM DIFFERENTIAL GEOMETRY OF AW( $k$-TYPE CURVES IN PSEUDO-GALILEAN 3-SPACE
}

\author{
M. KHALIFA SAAD ${ }^{1,2, *}$ AND H. S. ABDEL-AZIZ ${ }^{2}$ \\ ${ }^{1}$ Department of Mathematics, Faculty of Science, Islamic University of Madinah, 170 Madinah, KSA \\ ${ }^{2}$ Department of Mathematics, Faculty of Science, Sohag University, 82524 Sohag, Egypt \\ *Corresponding author: mohammed.khalifa@iu.edu.sa

\begin{abstract}
The aim of this paper is to study $\mathrm{AW}(k)$-type $(1 \leq k \leq 3)$ curves according to the equiform differential geometry of the pseudo-Galilean space $G_{3}^{1}$. We give some geometric properties of AW $(k)$ and weak AW $(k)$-type curves. Moreover, we give some relations between the equiform curvatures of these curves. Finally, examples of some special curves are given and plotted to support our main results.
\end{abstract}

\section{INTRODUCTION}

The geometry of space is associated with mathematical group. The idea of invariance of geometry under transformation group may imply that, on some spacetimes of maximum symmetry there should be a principle of relativity which requires the invariance of physical laws without gravity under transformations among inertial systems [1]. The theory of curves and the curves of constant curvature in the equiform differential geometry of the isotropic spaces $I_{3}^{1}, I_{3}^{2}$ and the Galilean space $G_{3}$ are described in [2] and [3], respectively. The pseudo-Galilean space is one of the real Cayley-Klein spaces. It has projective signature $(0,0,+,-)$ according to [2]. The absolute of the pseudo-Galilean space is an ordered triple $\{w, f, I\}$ where $w$ is the ideal plane, $f$ a line in $w$ and $I$ is the fixed hyperbolic involution of the points of $f$. In [4], from the differential

Received 2019-10-24; accepted 2019-11-20; published 2020-01-02.

2010 Mathematics Subject Classification. 53A04, 53A35, 53C40.

Key words and phrases. AW $(k)$-type curves; spacelike and timelike curves; general helix; equiform geometry; pseudo-Galilean space.

(C) 2020 Authors retain the copyrights of their papers, and all open access articles are distributed under the terms of the Creative Commons Attribution License. 
geometric point of view, K. Arslan and A. West defined the notion of AW(k)-type submanifolds. Since then, many works have been done related to AW(k)-type submanifolds (see, for example, [5-10]). In [9], Özgür and Gezgin studied a Bertrand curve of AW(k)-type and furthermore, they showed that there is no such Bertrand curve of $\mathrm{AW}(1)$ and $\mathrm{AW}(3)$-types if and only if it is a right circular helix. In addition, they studied weak $\mathrm{AW}(2)$-type and AW(3)-type conical geodesic curves in Euclidean 3-space $E^{3}$. Besides, In 3-dimensional Galilean space and Lorentz space, the curves of $\mathrm{AW}(k)$-type were investigated in $[6,8]$. In [7], the authors gave curvature conditions and characterizations related to $\mathrm{AW}(k)$-type curves in $E^{n}$ and in [10], the authors investigated curves of AW( $k$-type in the 3-dimensional null cone.

This paper is organized as follows. In section 2, the basic notions and properties of a pseudo-Galilean geometry are reviewed. In section 3, properties of the equiform geometry of the pseudo-Galilean space $G_{3}^{1}$ are given. Section 4 contains a study of AW $(k)$-type equiform Frenet curves. Finally, some examples of special curves in $G_{3}^{1}$ are included in section 5 .

\section{BASIC CONCEPTS}

In this section, we recall some basic notions from pseudo-Galilean geometry $[11,12]$. In the inhomogeneous affine coordinates for points and vectors (point pairs) the similarity group $H_{8}$ of $G_{3}^{1}$ has the following form

$$
\begin{aligned}
& \bar{x}=a+b \cdot x, \\
& \bar{y}=c+d \cdot x+r \cdot \cosh \theta \cdot y+r \cdot \sinh \theta \cdot z, \\
& \bar{z}=e+f \cdot x+r \cdot \sinh \theta \cdot y+r \cdot \cosh \theta \cdot z,
\end{aligned}
$$

where $a, b, c, d, e, f, r$ and $\theta$ are real numbers. Particularly, for $b=r=1$, the group (2.1) becomes the group $B_{6} \subset H_{8}$ of isometries (proper motions) of the pseudo-Galilean space $G_{3}^{1}$. The motion group leaves invariant the absolute figure and defines the other invariants of this geometry. It has the following form

$$
\begin{aligned}
& \bar{x}=a+x, \\
& \bar{y}=c+d \cdot x+\cosh \theta \cdot y+\sinh \theta \cdot z, \\
& \bar{z}=e+f \cdot x+\sinh \theta \cdot y+\cosh \theta \cdot z .
\end{aligned}
$$

According to the motion group in the pseudo-Galilean space, there are non-isotropic vectors $A\left(A_{1}, A_{2}, A_{3}\right)$ (for which holds $A_{1} \neq 0$ ) and four types of isotropic vectors: spacelike $\left(A_{1}=0, A_{2}^{2}-A_{3}^{2}>0\right.$ ), timelike $\left(A_{1}=0, A_{2}^{2}-A_{3}^{2}<0\right)$ and two types of lightlike vectors $\left(A_{1}=0, A_{2}= \pm A_{3}\right)$. The scalar product of two vectors $u=\left(u_{1}, u_{2}, u_{3}\right)$ and $v=\left(v_{1}, v_{2}, v_{3}\right)$ in $G_{3}^{1}$ is defined by

$$
\langle u, v\rangle=\left\{\begin{array}{cl}
u_{1} v_{1}, & \text { if } u_{1} \neq 0 \text { or } v_{1} \neq 0, \\
u_{2} v_{2}-u_{3} v_{3} & \text { if } u_{1}=0 \text { and } v_{1}=0 .
\end{array}\right.
$$


We introduce a pseudo-Galilean cross product in the following way

$$
u \times_{G_{3}^{1}} v=\left|\begin{array}{ccc}
0 & -j & k \\
u_{1} & u_{2} & u_{3} \\
v_{1} & v_{2} & v_{3}
\end{array}\right|,
$$

where $j=(0,1,0)$ and $k=(0,0,1)$ are unit spacelike and timelike vectors, respectively. Let us recall basic facts about curves in $G_{3}^{1}$, that were introduced in [13-15].

A curve $\gamma(s)=(x(s), y(s), z(s))$ is called an admissible curve if it has no inflection points $(\dot{\gamma} \times \ddot{\gamma} \neq 0)$ and no isotropic tangents $(\dot{x} \neq 0)$ or normals whose projections on the absolute plane would be lightlike vectors $(\dot{y} \neq \pm \dot{z})$. An admissible curve in $G_{3}^{1}$ is an analogue of a regular curve in Euclidean space [12].

For an admissible curve $\gamma(s): I \subseteq \mathbb{R} \rightarrow G_{3}^{1}$, the curvature $\kappa(s)$ and torsion $\tau(s)$ are defined by

$$
\kappa(s)=\frac{\sqrt{\left|\ddot{y}(s)^{2}-\ddot{z}(s)^{2}\right|}}{(\dot{x}(s))^{2}}, \tau(s)=\frac{\ddot{y}(s) \dddot{z}(s)-\dddot{y}(s) \ddot{z}(s)}{|\dot{x}(s)|^{5} \cdot \kappa^{2}(s)},
$$

expressed in components. Hence, for an admissible curve $\gamma: I \subseteq \mathbb{R} \rightarrow G_{3}^{1}$ parameterized by the arc length $s$ with differential form $d s=d x$ is given by

$$
\gamma(x)=(x, y(x), z(x))
$$

The formulas (2.3) have the following form

$$
\kappa(x)=\sqrt{\left|y^{\prime \prime}(x)^{2}-z^{\prime \prime}(x)^{2}\right|}, \tau(x)=\frac{y^{\prime \prime}(x) z^{\prime \prime \prime}(x)-y^{\prime \prime \prime}(x) z^{\prime \prime}(x)}{\kappa^{2}(x)} .
$$

The associated trihedron is given by

$$
\begin{aligned}
& \mathbf{e}_{1}=\gamma^{\prime}(x)=\left(1, y^{\prime}(x), z^{\prime}(x)\right) \\
& \mathbf{e}_{2}=\frac{1}{\kappa(x)} \gamma^{\prime \prime}(x)=\frac{1}{\kappa(x)}\left(0, y^{\prime \prime}(x), z^{\prime \prime}(x)\right), \\
& \mathbf{e}_{3}=\frac{1}{\kappa(x)}\left(0, \epsilon z^{\prime \prime}(x), \epsilon y^{\prime \prime}(x)\right)
\end{aligned}
$$

where $\epsilon=+1$ or $\epsilon=-1$, chosen by criterion $\operatorname{det}\left(e_{1}, e_{2}, e_{3}\right)=1$, that means

$$
\left|y^{\prime \prime}(x)^{2}-z^{\prime \prime}(x)^{2}\right|=\epsilon\left(y^{\prime \prime}(x)^{2}-z^{\prime \prime}(x)^{2}\right) .
$$

The curve $\gamma$ given by (2.4) is timelike (resp. spacelike) if $\mathbf{e}_{2}(s)$ is a spacelike (resp. timelike) vector. The principal normal vector or simply normal is spacelike if $\epsilon=+1$ and timelike if $\epsilon=-1$. For derivatives of the tangent $\mathbf{e}_{1}$, normal $\mathbf{e}_{2}$ and binormal $\mathbf{e}_{3}$ vector fields, the following Frenet formulas in $G_{3}^{1}$ hold:

$$
\begin{aligned}
& \mathbf{e}_{1}^{\prime}(x)=\kappa(x) \mathbf{e}_{2}(x), \\
& \mathbf{e}_{2}^{\prime}(x)=\tau(x) \mathbf{e}_{3}(x), \\
& \mathbf{e}_{3}^{\prime}(x)=\tau(x) \mathbf{e}_{2}(x) .
\end{aligned}
$$




\section{Frenet EQUations ACCORDing to the EQUiform GeOMETRY of $G_{3}^{1}$}

This section contains some important facts about equiform geometry. The equiform differential geometry of curves in the pseudo-Galilean space $G_{3}^{1}$ has been described in [11]. In the equiform geometry a few specific terms will be introduced. So, let $\gamma(s): I \rightarrow G_{3}^{1}$ be an admissible curve in the pseudo-Galilean space $G_{3}^{1}$, the equiform parameter of $\gamma$ is defined by

$$
\sigma:=\int \frac{1}{\rho} d s=\int \kappa d s
$$

where $\rho=\frac{1}{\kappa}$ is the radius of curvature of the curve $\gamma$. Then, we have

$$
\frac{d s}{d \sigma}=\rho .
$$

Let $h$ be a homothety with center at origin and the coefficient $\mu$. If we put $\bar{\gamma}=h(\gamma)$, then it follows

$$
\bar{s}=\mu s \text { and } \bar{\rho}=\mu \rho,
$$

where $\bar{s}$ is the arc-length parameter of $\bar{\gamma}$ and $\bar{\rho}$ is the radius of curvature of this curve. Therefore, $\sigma$ is an equiform invariant parameter of $\gamma$ (see [11]).

Notation 3.1. The functions $\kappa$ and $\tau$ are not invariants of the homothety group, then from (2.3) it follows that $\bar{\kappa}=\frac{1}{\mu} \kappa$ and $\bar{\tau}=\frac{1}{\mu} \tau$.

Now we define the Frenet formulas of the curve $\gamma$ with respect to its equiform invariant parameter $\sigma$ in $G_{3}^{1}$. The vector

$$
\mathbf{T}=\frac{d \gamma}{d \sigma}
$$

is called a tangent vector of the curve $\gamma$. From (2.6) and (3.1), we get

$$
\mathbf{T}=\frac{d \gamma}{d s} \frac{d s}{d \sigma}=\rho \cdot \frac{d \gamma}{d s}=\rho \cdot \mathbf{e}_{1} .
$$

Also, the principal normal and the binormal vectors are respectively, given by

$$
\mathbf{N}=\rho \cdot \mathbf{e}_{2}, \quad \mathbf{B}=\rho \cdot \mathbf{e}_{3} .
$$

It is easy to show that $\{\mathbf{T}, \mathbf{N}, \mathbf{B}\}$ is an equiform invariant frame of $\gamma$. On the other hand, the derivatives of these vectors with respect to $\sigma$ are given by

$$
\left[\begin{array}{c}
\mathbf{T} \\
\mathbf{N} \\
\mathbf{B}
\end{array}\right]^{\prime}=\left[\begin{array}{ccc}
\dot{\rho} & 1 & 0 \\
0 & \dot{\rho} & \rho \tau \\
0 & \rho \tau & \dot{\rho}
\end{array}\right]\left[\begin{array}{c}
\mathbf{T} \\
\mathbf{N} \\
\mathbf{B}
\end{array}\right]
$$

The functions $\mathcal{K}: I \rightarrow \mathbb{R}$ defined by $\mathcal{K}=\dot{\rho}$ is called the equiform curvature of the curve $\gamma$ and $\mathcal{T}: I \rightarrow \mathbb{R}$ defined by $\mathcal{T}=\rho \tau=\frac{\tau}{\kappa}$ is called the equiform torsion of this curve. In the light of this, the formulas (3.4) 
analogous to the Frenet formulas in the equiform geometry of the pseudo-Galilean space $G_{3}^{1}$ can be written as

$$
\left[\begin{array}{c}
\mathbf{T} \\
\mathbf{N} \\
\mathbf{B}
\end{array}\right]^{\prime}=\left[\begin{array}{ccc}
\mathcal{K} & 1 & 0 \\
0 & \mathcal{K} & \mathcal{T} \\
0 & \mathcal{T} & \mathcal{K}
\end{array}\right]\left[\begin{array}{c}
\mathbf{T} \\
\mathbf{N} \\
\mathbf{B}
\end{array}\right]
$$

The equiform parameter $\sigma=\int \kappa(s) d s$ for closed curves is called the total curvature, and it plays an important role in global differential geometry of Euclidean space. Also, the function $\frac{\tau}{\kappa}$ has been already known as a conical curvature and it also has interesting geometric interpretation.

Notation 3.2. Let $\gamma: I \rightarrow G_{3}^{1}$ be a Frenet curve in the equiform geometry of $G_{3}^{1}$, the following statements are true ( for more details, see [11,13]):

(1) If $\gamma(s)$ is an isotropic logarithmic spiral in $G_{3}^{1}$. Then, $\mathcal{K}=$ const. $\neq 0$ and $\mathcal{T}=0$,

(2) If $\gamma(s)$ is a circular helix in $G_{3}^{1}$. Then, $\mathcal{K}=0$ and $\mathcal{T}=$ const. $\neq 0$,

(3) If $\gamma(s)$ is an isotropic circle in $G_{3}^{1}$. Then, $\mathcal{K}=0$ and $\mathcal{T}=0$.

\section{AW( $k$-Type CURVES In THE EQUiform GEOMETRY of $G_{3}^{1}$}

Let $\gamma(s): I \rightarrow G_{3}^{1}$ be a curve in the equiform geometry of the pseudo-Galilean space $G_{3}^{1}$. The curve $\gamma$ is called a Frenet curve of osculating order $l$ if its derivatives:

$$
\gamma^{\prime}(s), \gamma^{\prime \prime}(s), \gamma^{\prime \prime \prime}(s), \ldots, \gamma^{(l)}(s)
$$

are linearly dependent and

$$
\gamma^{\prime}(s), \gamma^{\prime \prime}(s), \gamma^{\prime \prime \prime}(s), \ldots, \gamma^{(l+1)}(s),
$$

are no longer linearly independent for all $s \in I$.

To each Frenet curve of order 3, one can associate an orthonormal 3-frame $\{\mathbf{T}, \mathbf{N}, \mathbf{B}\}$ along $\gamma$, such that $\gamma^{\prime}(s)=\frac{1}{\rho} \mathbf{T}$, called the equiform Frenet frame (Eqs. (3.5)).

Now, we consider equiform Frenet curves of osculating order 3 in $G_{3}^{1}$ and discuss some important results.

Let $\gamma(s): I \rightarrow G_{3}^{1}$ be a Frenet curve in the equiform geometry of the pseudo-Galilean space. By the use of Frenet formulas (3.5), we obtain the higher order derivatives of $\gamma$ as follows

$$
\begin{aligned}
\gamma^{\prime}(s) & =\frac{d \gamma}{d \sigma} \frac{d \sigma}{d s}=\frac{1}{\rho} \mathbf{T}, \\
\gamma^{\prime \prime}(s) & =\frac{1}{\rho^{2}} \mathbf{N}, \\
\gamma^{\prime \prime \prime}(s) & =\frac{1}{\rho^{3}}(-\mathcal{K} \mathbf{N}+\mathcal{T} \mathbf{B}), \\
\gamma^{\prime \prime \prime \prime}(s) & =\frac{1}{\rho^{4}}\left[\left(2 \mathcal{K}^{2}+\mathcal{T}^{2}-\mathcal{K}^{\prime}\right) \mathbf{N}+\left(\mathcal{T}^{\prime}-3 \mathcal{K} \mathcal{T}\right) \mathbf{B}\right] .
\end{aligned}
$$


Notation 4.1. Let us write

$$
\begin{aligned}
Q_{1} & =\frac{1}{\rho^{2}} \mathbf{N} \\
Q_{2} & =\frac{1}{\rho^{3}}(-\mathcal{K} \mathbf{N}+\mathcal{T} \mathbf{B}), \\
Q_{3} & =\frac{1}{\rho^{4}}\left[\left(2 \mathcal{K}^{2}+\mathcal{T}^{2}-\mathcal{K}^{\prime}\right) \mathbf{N}+\left(\mathcal{T}^{\prime}-3 \mathcal{K} \mathcal{T}\right) \mathbf{B}\right] .
\end{aligned}
$$

Notation 4.2. $\gamma^{\prime}(s), \gamma^{\prime \prime}(s), \gamma^{\prime \prime \prime}(s)$ and $\gamma^{\prime \prime \prime \prime}(s)$ are linearly dependent if and only if $Q_{1}, Q_{2}$ and $Q_{3}$ are linearly dependent.

Definition 4.1. [5] Frenet curves (of osculating order 3) in the equiform geometry of the pseudo-Galilean space $G_{3}^{1}$ are called curves of type:

(1) equiform $A W(1)$ if they satisfy $Q_{3}=0$,

(2) equiform $A W(2)$ if they satisfy $\left\|Q_{2}\right\|^{2} Q_{3}=\left\langle Q_{3}, Q_{2}\right\rangle Q_{2}$,

(3) equiform $A W(3)$ if they satisfy $\left\|Q_{1}\right\|^{2} Q_{3}=\left\langle Q_{3}, Q_{1}\right\rangle Q_{1}$,

(4) weak equiform $A W(2)$ if they satisfy

$$
Q_{3}=\left\langle Q_{3}, Q_{2}^{*}\right\rangle Q_{2}^{*}
$$

(5) weak equiform $A W(3)$ if they satisfy

$$
Q_{3}=\left\langle Q_{3}, Q_{1}^{*}\right\rangle Q_{1}^{*}
$$

where

$$
\begin{aligned}
Q_{1}^{*} & =\frac{Q_{1}}{\left\|Q_{1}\right\|}, \\
Q_{2}^{*} & =\frac{Q_{2}-\left\langle Q_{2}, Q_{1}^{*}\right\rangle Q_{1}^{*}}{\left\|Q_{2}-\left\langle Q_{2}, Q_{1}^{*}\right\rangle Q_{1}^{*}\right\|} .
\end{aligned}
$$

Proposition 4.1. Let $\gamma: I \rightarrow G_{3}^{1}$ be a Frenet curve (of osculating order 3 ) in the equiform geometry of the pseudo-Galilean space $G_{3}^{1}$, therefore

(i) $\gamma$ is of type weak equiform $A W(2)$ if and only if

$$
2 \mathcal{K}^{2}+\mathcal{T}^{2}-\mathcal{K}^{\prime}=0
$$

(ii) $\gamma$ is of type weak equiform $A W(3)$ if and only if

$$
\mathcal{T}^{\prime}-3 \mathcal{K} \mathcal{T}(s)=0
$$

Proof. Using Definition 4.1 and Notation 4.1, the proof will be obvious. 
Theorem 4.1. Let $\gamma: I \rightarrow G_{3}^{1}$ be a Frenet curve (of osculating order 3 ) in the equiform geometry of the pseudo-Galilean space $G_{3}^{1}$. Then $\gamma$ is of type equiform $A W(1)$ if and only if

$$
-\mathcal{K}^{\prime}+2 \mathcal{K}^{2}+\mathcal{T}^{2}=0
$$

$$
3 \mathcal{K} \mathcal{T}-\mathcal{T}^{\prime}=0
$$

Proof. Since $\gamma$ is of type equiform AW(1), then from (4.3), we obtain

$$
\frac{1}{\rho^{4}}\left[\left(2 \mathcal{K}^{2}+\mathcal{T}^{2}(s)-\mathcal{K}^{\prime}\right) \mathbf{N}+\left(\mathcal{T}^{\prime}-3 \mathcal{K} \mathcal{T}\right) \mathbf{B}\right]=0
$$

As we know, the vectors $\mathbf{N}$ and $\mathbf{B}$ are linearly independent, so we can write

$$
2 \mathcal{K}^{2}+\mathcal{T}^{2}-\mathcal{K}^{\prime}=0 \text { and } \mathcal{T}^{\prime}-3 \mathcal{K} \mathcal{T}=0
$$

The converse statement is straightforward and therefore, the proof is completed.

Theorem 4.2. Let $\gamma: I \rightarrow G_{3}^{1}$ be a Frenet curve (of osculating order 3 ) in the equiform geometry of the pseudo-Galilean space $G_{3}^{1}$. Then $\gamma$ is of type equiform $A W(2)$ if

$$
\mathcal{K}^{2} \mathcal{T}-\mathcal{K} \mathcal{T}^{\prime}+\mathcal{T} \mathcal{K}^{\prime}-\mathcal{T}^{3}=0
$$

Proof. Assuming that $\gamma$ is a Frenet curve in the equiform geometry of $G_{3}^{1}$, then from (4.2) and (4.3), one can write

$$
\begin{aligned}
& Q_{2}=a_{11} \mathbf{N}+a_{12} \mathbf{B}, \\
& Q_{3}=a_{21} \mathbf{N}+a_{22} \mathbf{B},
\end{aligned}
$$

where $a_{11}, a_{12}, a_{21}$ and $a_{22}$ are differentiable functions. Since $Q_{2}$ and $Q_{3}$ are linearly dependent, hence coefficients determinant equals zero, that is

$$
\left|\begin{array}{ll}
a_{11} & a_{12} \\
a_{21} & a_{22}
\end{array}\right|=0,
$$

where

$$
\begin{aligned}
a_{11} & =\frac{-1}{\rho^{3}} \mathcal{K}, a_{12}=\frac{1}{\rho^{3}} \mathcal{T} \\
a_{21} & =\frac{1}{\rho^{4}}\left[-\mathcal{K}^{\prime}+2 \mathcal{K}^{2}+\mathcal{T}^{2}\right] \\
a_{22} & =\frac{1}{\rho^{4}}\left[-3 \mathcal{K} \mathcal{T}+\mathcal{T}^{\prime}\right]
\end{aligned}
$$

From (4.11) and (4.12), we obtain (4.10). 
Theorem 4.3. Let $\gamma: I \rightarrow G_{3}^{1}$ be a Frenet curve (of osculating order 3 ) in the equiform geometry of $G_{3}^{1}$. Then $\gamma$ is of equiform $A W(3)$-type if

$$
\mathcal{T}^{\prime}-3 \mathcal{K} \mathcal{T}=0
$$

Proof. Using Definition 4.1 and Eqs. (4.1) and (4.3), we obtain (4.13).

\section{Computational examples}

We consider some examples (timelike and spacelike curves $[11,12]$ ) which characterize equiform general (circular) helices with respect to the Frenet frame $\{\mathbf{T}, \mathbf{N}, \mathbf{B}\}$ in the equiform geometry of $G_{3}^{1}$ which satisfy some conditions of equiform curvatures (i) $\mathcal{K}=\mathcal{K}(s), \mathcal{T}=\mathcal{T}(s)$ (ii) $\mathcal{K}=$ const. $\neq 0, \mathcal{T}=$ const. $\neq 0$ (iii) $\mathcal{K}=$ const. $\neq 0, \mathcal{T}=0$.

Example 5.1. Consider the equiform timelike general helix $\mathbf{r}: I \longrightarrow G_{3}^{1}, I \subseteq \mathbb{R}$ which parameterized by the arc length $s$ with differential form $d s=d x$ is given by

$$
\mathbf{r}(x)=(x, y(x), z(x))
$$

where

$$
\begin{aligned}
x(s) & =s, \\
y(s) & =\frac{e^{-a s}}{\left(a^{2}-b^{2}\right)^{2}}\left(\left(a^{2}+b^{2}\right) \cosh (b s)+2 a b \sinh (b s)\right), \\
z(s) & =\frac{e^{-a s}}{\left(a^{2}-b^{2}\right)^{2}}\left(2 a b \cosh (b s)+\left(a^{2}+b^{2}\right) \sinh (b s)\right) ; \\
a, b & \in \mathbb{R}-\{0\} .
\end{aligned}
$$

The corresponding derivatives of $\mathbf{r}$ are as follows

$$
\begin{aligned}
\mathbf{r}^{\prime} & =\left(1, \frac{-e^{-a s}}{\left(a^{2}-b^{2}\right)}(a \cosh (b s)+b \sinh (b s)), \frac{e^{-a s}}{\left(b^{2}-a^{2}\right)}(b \cosh (b s)+a \sinh (b s))\right), \\
\mathbf{r}^{\prime \prime} & =\left(0, e^{-a s} \cosh (b s), e^{-a s} \sinh (b s)\right) \\
\mathbf{r}^{\prime \prime \prime} & =\left(0, e^{-a s}(-a \cosh (b s)+b \sinh (b s)), e^{-a s}(b \cosh (b s)-a \sinh (b s))\right) .
\end{aligned}
$$

The tangent vector of $\mathbf{r}$ has the form

$$
\begin{aligned}
\mathbf{e}_{1} & =\left(x^{\prime}, y^{\prime}, z^{\prime}\right) \\
& =\left(1, \frac{-e^{-a s}}{\left(a^{2}-b^{2}\right)}(a \cosh (b s)+b \sinh (b s)), \frac{e^{-a s}}{\left(b^{2}-a^{2}\right)}(b \cosh (b s)+a \sinh (b s))\right),
\end{aligned}
$$

and the two normals (normal and binormal) of the curve are, respectively

$$
\begin{aligned}
& \mathbf{e}_{2}=(0, \cosh (b s), \sinh (b s)) \\
& \mathbf{e}_{3}=(0, \sinh (b s), \cosh (b s)) ; \operatorname{det}\left[\mathbf{e}_{1}, \mathbf{e}_{2}, \mathbf{e}_{3}\right]=1 .
\end{aligned}
$$


Therefore, the curvature and torsion of $\mathbf{r}$ are respectively, given by

$$
\kappa=e^{-a s}, \tau=b
$$

From the equiform Frenet formulas, we can express the vector fields $\mathbf{T}, \mathbf{N}, \mathbf{B}$ as follows

$$
\begin{aligned}
& \mathbf{T}=\left(e^{a s}, \frac{-1}{\left(a^{2}-b^{2}\right)}(a \cosh (b s)+b \sinh (b s)), \frac{1}{\left(b^{2}-a^{2}\right)}(b \cosh (b s)+a \sinh (b s))\right), \\
& \mathbf{N}=\left(0, e^{a s} \cosh (b s), e^{a s} \sinh (b s)\right), \\
& \mathbf{B}=\left(0, e^{a s} \sinh (b s), e^{a s} \cosh (b s)\right),
\end{aligned}
$$

respectively. In the light of this, the equiform curvatures are given by

$$
\mathcal{K}=a e^{a s}, \mathcal{T}=-b e^{a s}
$$

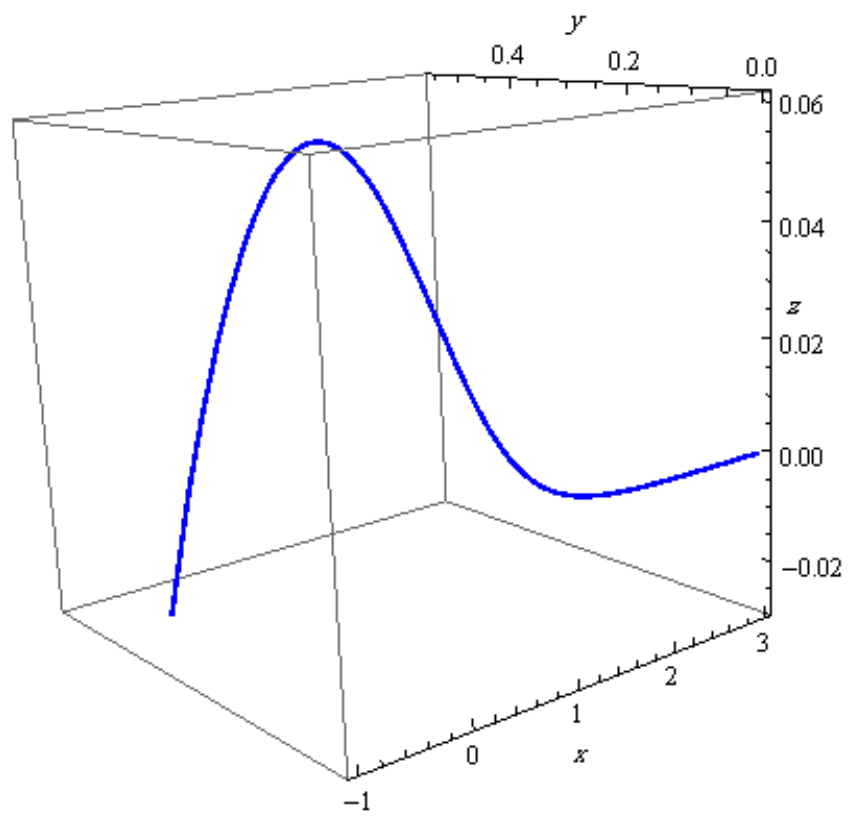

Figure 1. Equiform timelike general helix with $\mathcal{K}=5 e^{5 s}, \mathcal{T}=-2 e^{5 s}$.

Example 5.2. Let $\mathbf{r}: I \longrightarrow G_{3}^{1}, I \subseteq \mathbb{R}$ be the equiform spacelike general helix, and it is given by

$$
\mathbf{r}(x)=(x, y(x), z(x))
$$


where

$$
\begin{aligned}
x(s) & =s, \\
y(s) & =\frac{e^{-a s}}{\left(a^{2}-b^{2}\right)^{2}}\left(2 a b \cosh (b s)+\left(a^{2}+b^{2}\right) \sinh (b s)\right), \\
z(s) & =\frac{e^{-a s}}{\left(a^{2}-b^{2}\right)^{2}}\left(\left(a^{2}+b^{2}\right) \cosh (b s)+2 a b \sinh (b s)\right) ; \\
a, b & \in \mathbb{R}-\{0\} .
\end{aligned}
$$

For the coordinate functions of $\mathbf{r}$, we have

$$
\begin{aligned}
\mathbf{r}^{\prime} & =\left(1, \frac{e^{-a s}}{\left(b^{2}-a^{2}\right)}(b \cosh (b s)+a \sinh (b s)), \frac{-e^{-a s}}{\left(a^{2}-b^{2}\right)}(a \cosh (b s)+b \sinh (b s))\right), \\
\mathbf{r}^{\prime \prime} & =\left(0, e^{-a s} \sinh (b s), e^{-a s} \cosh (b s)\right), \\
\mathbf{r}^{\prime \prime \prime} & =\left(0, e^{-a s}(b \cosh (b s)-a \sinh (b s)), e^{-a s}(b \sinh (b s)-a \cosh (b s))\right) .
\end{aligned}
$$

Also, the associated trihedron is given by

$$
\begin{aligned}
& \mathbf{e}_{1}=\left(1, \frac{e^{-a s}}{\left(b^{2}-a^{2}\right)}(b \cosh (b s)+a \sinh (b s)), \frac{-e^{-a s}}{\left(a^{2}-b^{2}\right)}(a \cosh (b s)+b \sinh (b s))\right), \\
& \mathbf{e}_{2}=(0, \sinh (b s), \cosh (b s)), \\
& \mathbf{e}_{3}=(0,-\cosh (b s),-\sinh (b s)) .
\end{aligned}
$$

The curvature and torsion of this curve are

$$
\kappa=e^{-a s}, \tau=-b .
$$

Furthermore, the tangent, normal and binormal vector fields in the equiform geometry of $G_{3}^{1}$ are obtained as follows

$$
\begin{aligned}
& \mathbf{T}=\left(e^{a s}, \frac{1}{\left(b^{2}-a^{2}\right)}(b \cosh (b s)+a \sinh (b s)), \frac{-1}{\left(a^{2}-b^{2}\right)}(a \cosh (b s)+b \sinh (b s))\right), \\
& \mathbf{N}=\left(0, e^{a s} \sinh (b s), e^{a s} \cosh (b s)\right), \\
& \mathbf{B}=\left(0,-e^{a s} \cosh (b s),-e^{a s} \sinh (b s)\right),
\end{aligned}
$$

respectively.

The equiform curvatures of $\mathbf{r}$ are

$$
\mathcal{K}=a e^{a s}, \mathcal{T}=-b e^{a s}
$$

Example 5.3. Consider the equiform timelike circular helix $\mathbf{r}: I \longrightarrow G_{3}^{1}, I \subseteq \mathbb{R}$ is given by

$$
\mathbf{r}(x)=(x, y(x), z(x))
$$




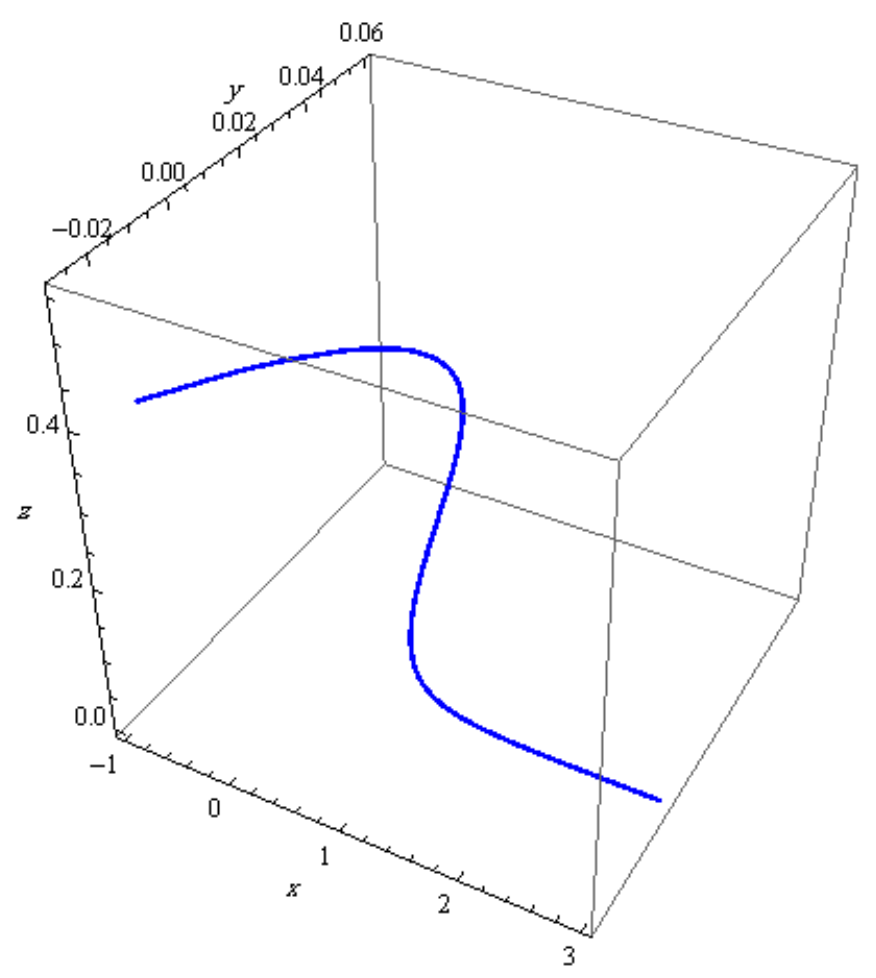

Figure 2. Equiform spacelike general helix with $\mathcal{K}=5 e^{5 s}, \mathcal{T}=-2 e^{5 s}$.

where

$$
\begin{aligned}
x(s) & =s, \\
y(s) & =\frac{a^{3} s}{b\left(b^{2}-a^{2}\right)}\left(b \sinh \left(\frac{b}{a} \ln (a s)\right)-a \cosh \left(\frac{b}{a} \ln (a s)\right)\right), \\
z(s) & =\frac{a^{3} s}{b\left(b^{2}-a^{2}\right)}\left(b \cosh \left(\frac{b}{a} \ln (a s)\right)-a \sinh \left(\frac{b}{a} \ln (a s)\right)\right) ; \\
a, b & \in \mathbb{R}-\{0\} .
\end{aligned}
$$

For this curve, the equiform vector fields are obtained as follows

$$
\begin{aligned}
\mathbf{T} & =\left(\frac{s}{a}, \frac{a s}{b} \cosh \left(\frac{b}{a} \ln (a s)\right), \frac{a s}{b} \sinh \left(\frac{b}{a} \ln (a s)\right)\right), \\
\mathbf{N} & =\left(0, \frac{s}{a} \sinh \left(\frac{b}{a} \ln (a s)\right), \frac{s}{a} \cosh \left(\frac{b}{a} \ln (a s)\right)\right), \\
\mathbf{B} & =\left(0, \frac{s}{a} \cosh \left(\frac{b}{a} \ln (a s)\right), \frac{s}{a} \sinh \left(\frac{b}{a} \ln (a s)\right)\right),
\end{aligned}
$$

respectively.

It follows that

$$
\mathcal{K}=\frac{1}{a}, \mathcal{T}=\frac{-b}{a^{2}}
$$




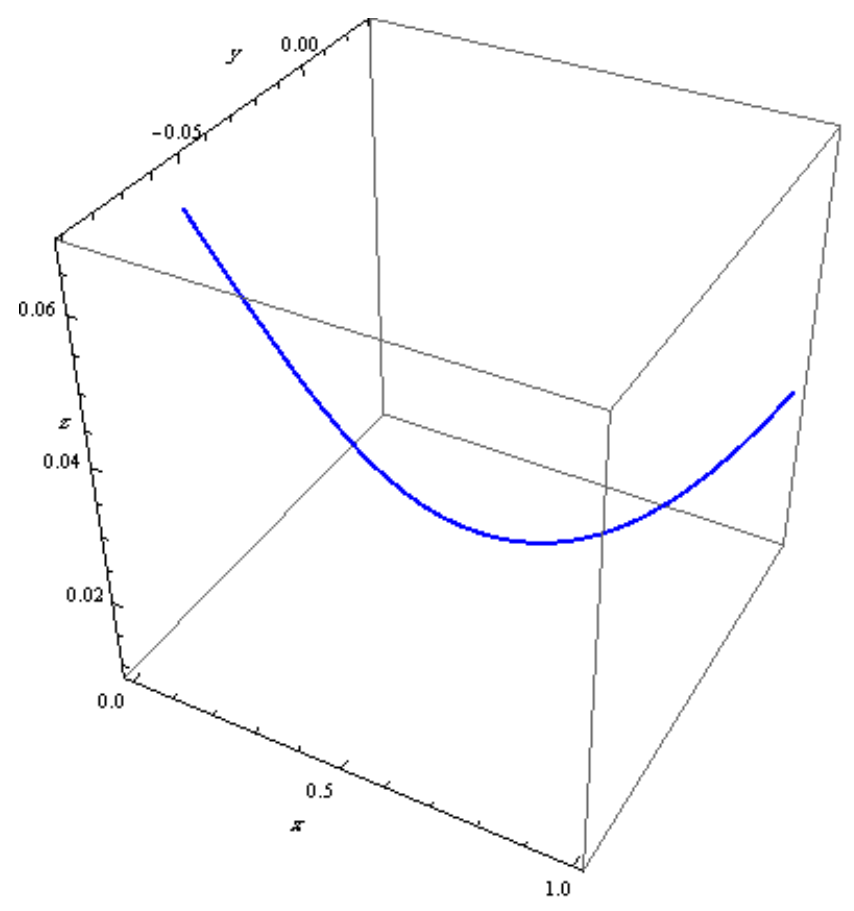

Figure 3. Equiform timelike circular helix with $\mathcal{K}=\frac{1}{2}, \mathcal{T}=\frac{-5}{4}$.

Example 5.4. Let the equiform spacelike circular helix $\mathbf{r}: I \longrightarrow G_{3}^{1}, I \subseteq \mathbb{R}$ be

$$
\mathbf{r}(x)=(x, y(x), z(x))
$$

where

$$
\begin{aligned}
x(s) & =s, \\
y(s) & =\frac{a^{3} s}{b\left(b^{2}-a^{2}\right)}\left(b \cosh \left(\frac{b}{a} \ln (a s)\right)-a \sinh \left(\frac{b}{a} \ln (a s)\right)\right), \\
z(s) & =\frac{a^{3} s}{b\left(b^{2}-a^{2}\right)}\left(b \sinh \left(\frac{b}{a} \ln (a s)\right)-a \cosh \left(\frac{b}{a} \ln (a s)\right)\right) ; \\
a, b & \in \mathbb{R}-\{0\} .
\end{aligned}
$$

Here, the equiform differential vectors respectively, are as follows

$$
\begin{aligned}
\mathbf{T} & =\left(\frac{s}{a}, \frac{a s}{b} \sinh \left(\frac{b}{a} \ln (a s)\right), \frac{a s}{b} \cosh \left(\frac{b}{a} \ln (a s)\right)\right), \\
\mathbf{N} & =\left(0, \frac{s}{a} \cosh \left(\frac{b}{a} \ln (a s)\right), \frac{s}{a} \sinh \left(\frac{b}{a} \ln (a s)\right)\right), \\
\mathbf{B} & =\left(0,-\frac{s}{a} \sinh \left(\frac{b}{a} \ln (a s)\right),-\frac{s}{a} \cosh \left(\frac{b}{a} \ln (a s)\right)\right) .
\end{aligned}
$$

Equiform curvature and equiform torsion are calculated as follows

$$
\mathcal{K}=\frac{1}{a}, \mathcal{T}=\frac{b}{a^{2}}
$$




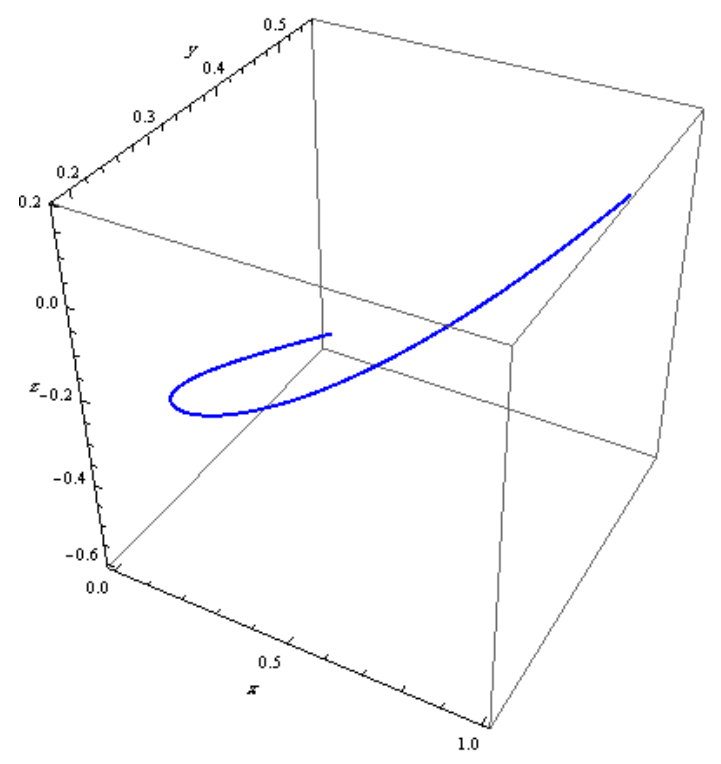

FigurE 4. Equiform spacelike circular helix with $\mathcal{K}=\frac{1}{3}, \mathcal{T}=\frac{4}{9}$.

Example 5.5. Let $\mathbf{r}: I \longrightarrow G_{3}^{1}, I \subseteq \mathbb{R}$ be a equiform timelike isotropic logarithmic spiral which parameterized by the arc length $s$ with differential form $d s=d x$, and is given by

$$
\mathbf{r}(x)=(x, y(x), 0)
$$

where

$$
\begin{aligned}
x(s) & =s, \\
y(s) & =\frac{a s+b}{a^{2}}(\ln (a s+b)-1), \\
z(s) & =0 ; \\
a, b & \in \mathbb{R}-\{0\} .
\end{aligned}
$$

For this curve, we get

$$
\begin{aligned}
\mathbf{r}^{\prime} & =\left(1, \frac{\ln (a s+b)}{a}, 0\right) \\
\mathbf{r}^{\prime \prime} & =\left(0, \frac{1}{a s+b}, 0\right) \\
\mathbf{r}^{\prime \prime \prime} & =\left(0, \frac{-a}{(a s+b)^{2}}, 0\right),
\end{aligned}
$$


and

$$
\begin{aligned}
& \mathbf{e}_{1}=\left(1, \frac{\ln (a s+b)}{a}, 0\right), \\
& \mathbf{e}_{2}=(0,1,0), \\
& \mathbf{e}_{3}=(0,0,1) ; \kappa=\frac{1}{a s+b}, \tau=0 .
\end{aligned}
$$

In this case, equiform Frenet vectors and equiform curvatures are as follows

$$
\begin{aligned}
& \mathbf{T}=\left(a s+b, \frac{(a s+b) \ln (a s+b)}{a}, 0\right), \\
& \mathbf{N}=(0, a s+b, 0), \\
& \mathbf{B}=(0,0, a s+b), \mathcal{K}=a, \mathcal{T}=0 .
\end{aligned}
$$

respectively.

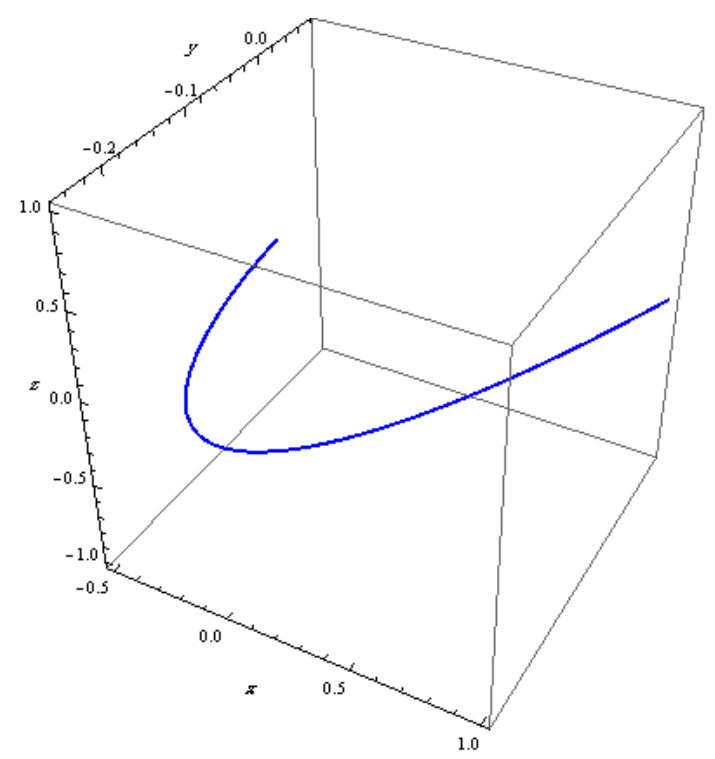

FIGURE 5. Equiform timelike isotropic logarithmic spiral with $\mathcal{K}=2, \mathcal{T}=0$.

From aforementioned calculations, according to (Proposition 4.2 and Theorems $4.1-4.3$ ), the first four examples are not characterize curves of equiform $\mathrm{AW}(1)$, weak equiform $\mathrm{AW}(2)$ or weak equiform $\mathrm{AW}(3)$ types. On the other hand, the last example shows that the curve is of equiform AW(2) and AW(3)-types and it is not of equiform AW(1)-type. Also, this curve is of weak equiform $\mathrm{AW}(2)$ and not of weak equiform $\mathrm{AW}(3)-$ types. 


\section{Conclusion}

In this paper, we have considered some special curves of equiform AW(k)-type of the pseudo-Galilean 3space. Also, using the equiform curvature conditions of these curves, the necessary and sufficient conditions for them to be equiform $\mathrm{AW}(k)$ and weak equiform $\mathrm{AW}(k)$-types are obtained. Furthermore, some examples to support our main results are given and plotted.

\section{ACKNOWLEDGMENT}

This research was supported by Islamic University of Madinah. We would like to thank our colleagues from Deanship of Scientific Research who provided insight and expertise that greatly assisted the research.

Conflicts of Interest: The author(s) declare that there are no conflicts of interest regarding the publication of this paper.

\section{REFERENCES}

[1] I. Yaglom, A simple non-Euclidean geometry and its physical basis, Springer-Verlag, in New York, 1979.

[2] B. J. Pavković, Equiform geometry of curves in the isotropic spaces $I_{3}^{1}$ and $I_{3}^{2}$, Rad JAZU, 1986, 39-44.

[3] B. J. Pavković and I. Kamenarović, The equiform differential geometry of curves in the Galilean space $G_{3}$, Glasnik Mat. 22 (42) (1987), 449-457.

[4] K. Arslan and A. West, Product submanifolds with pointwise 3-planar normal sections, Glasgow Math. J. 37 (1) (1995), 73-81.

[5] K. Arslan and C. Özgür, Curves and surfaces of AW(k)-type, Geometry and topology of submanifolds IX, World Scientific, 1999, 21-26.

[6] M. Külahci, M. Bektas and M. Ergüt, On harmonic curvatures of null curves of the AW(k)-type in Lorentzian space, Z. Naturforsch. A, 63 (5-6) (2008), 248-252.

[7] M. Külahci and M. Ergüt, Bertrand curves of AW(k)-type in Lorentzian space, Nonlinear Anal., Theory Methods Appl. 70 (2009), 1725-1731.

[8] M. Külahci, A.O. Öğrenmiş and M. Ergüt, New characterizations of curves in the Galilean space $G_{3}$, Int. J. Phys. Math. Sci. 1 (2010), 49-57.

[9] C. Özgür and F. Gezgin, On some curves of AW(k)-type, Differ. Geom. Dyn. Syst. 7 (2005), 74-80.

[10] D. W. Yoon, General Helices of AW(k)-Type in the Lie Group, J. Appl. Math. 2012 (2012), Article ID 535123.

[11] Z. Erjavec and B. Divjak, The equiform differential geometry of curves in the pseudo-Galilean space, Math. Commun. 13 (2008), 321-332.

[12] Z. Erjavec, On generalization of helices in the Galilean and the pseudo-Galilean space, J. Math. Res. 6 (3) (2014), 39-50.

[13] B. Divjak, The general solution of the Frenet's system of differential equations for curves in the pseudo-Galilean space $G_{3}^{1}$, Math. Commun. 2 (1997), 143-147.

[14] B. Divjak, Geometrija pseudogalilejevih prostora, Ph. D. thesis, University of Zagreb, 1997.

[15] B. Divjak, Curves in pseudo-Galilean geometry, Ann. Univ. Sci. Budapest. Sect. Math. 41 (1998), 117-128. 